\title{
MODELO CINEMÁTICO DE UN ROBOT HEXÁPODO CON "C-LEGS"
}

\author{
Jesús Tordesillas Torres \\ CAR UPM-CSIC, j.tordesillas@alumnos.upm.es
}

\author{
Jorge De León Rivas, Jaime Del Cerro, Antonio Barrientos \\ CAR UPM-CSIC, jorge.deleon, j.cerro, antonio.barrientos@upm.es
}

\section{Resumen}

En este artículo se profundiza en el modelado matemático de la odometría para robots hexápodos con extremidades denominadas C-legs. El estudio no es trivial, y se analizan todas las posibilidades que puede tener el sistema según las variables que se definan. Todo el estudio se ve reforzado con una serie de simulaciones realizadas donde los resultados obtenidos coinciden con los esperados.

Palabras clave: Robots hexápodos, C-legs, odometría

\section{ESTADO DEL ARTE}

La vigilancia, el mapeo de zonas, el acceso a zonas radiactivas, y la búsqueda de víctimas, son algunas de las muchas tareas en las que se está haciendo imprescindible el uso de los robots. El factor común a todas estas tareas, es el hecho de que el robot debe moverse por determinados terrenos abruptos y con desniveles.

Por esta razón, el diseño de sistemas de locomoción para estos robots móviles es el factor decisivo a la hora de construir un robot que pueda andar por este tipo de terrenos.

El empleo de orugas o ruedas son ineficientes para terrenos muy abruptos o con grandes diferencias de altura. Por ello, los ingenieros han empezado a imitar la evolución de la naturaleza en sus desarrollos, lo que se conoce como bioinspiración.

De todos los robots desarrollados mediante bioinspiración, un alto porcentaje están basados en la fisionomía de los hexápodos. Los hexápodos es la clasificación de artrópodos que más especies agrupa, incluyendo en ellos a los insectos. Éstos, a su vez se pueden clasificar según su sistema de locomoción sea terrestre o aéreo. Sin embargo, los robots bioinspirados suelen imitar a los de locomoción terrestre debido a que su adaptación al ámbito de la ingeniería es más simple.

Realizando un estudio de robots bioinspirados en hexápodos terrestres podemos encontrar a la generación LAURON, desarrollado por el FZI alemán, figura 1a [1]. Este robot está dotado de diferentes modos de marcha y es capaz de mantener la estabilidad incluso en terrenos con numerosos obstáculos. Otro ejemplo es el robot DIGbot, diseñado por la Case Western Reserve University y cuyo diseño de patas hace posible que pueda avanzar por troncos de árboles y paredes verticales, figura 1b [5].

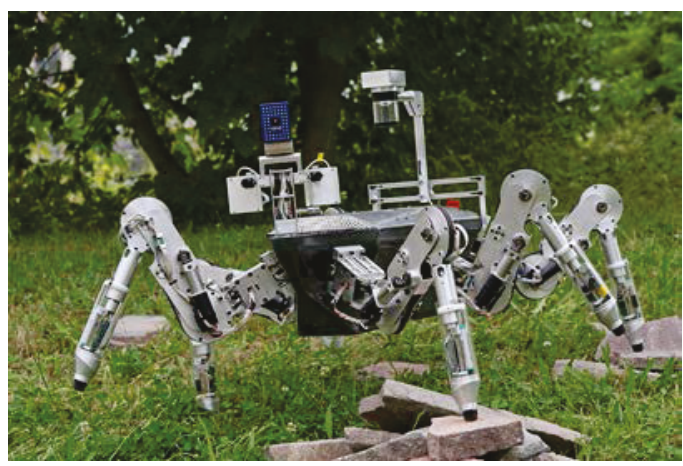

(a) Robot LAURON IV

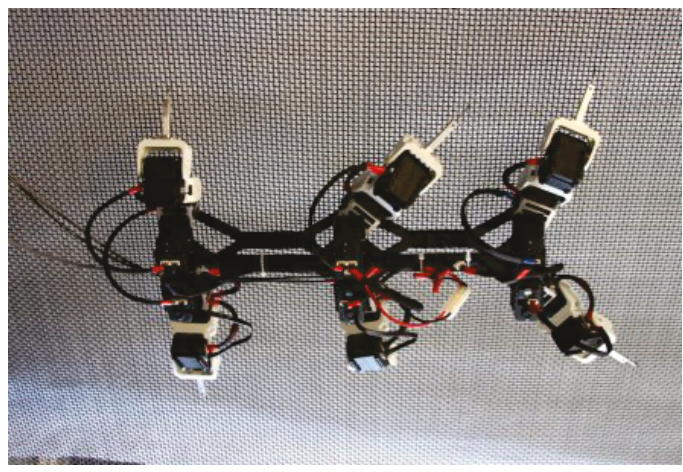

(b) Robot DIGbot

Figura 1: Ejemplos de robots hexápodos

Pero a pesar de que la cinemática de este tipo de robots está muy estudiada y resulta muy sencilla de implementar, la implementación mecánica y su control es muy complejo [4], por ello, se han desarrollado una nueva serie de robots que siguen siendo hexápodos pero presentan una nueva fisionomía en sus extremidades.

Estas nuevas extremidades tienen forma de letra $C$ y permiten a este tipo de robots conquistar, con unas velocidades mucho mayores y unos requisitos mecánicos menores, los territorios ya conquistados 
por los robots hexápodos que imitan fielmente a los insectos [3]. Una ventaja de este tipo de robots es, entre otras, su capacidad de lo que se conoce en la literatura como "locomoción sin patas", por la cual los robots pueden superar obstáculos cuando ninguna de sus patas toca el suelo [2].

Un robot que describe este tipo de diseño es el RHex desarrollado bajo la financiación del DARPA en Estados Unidos, figura 2.

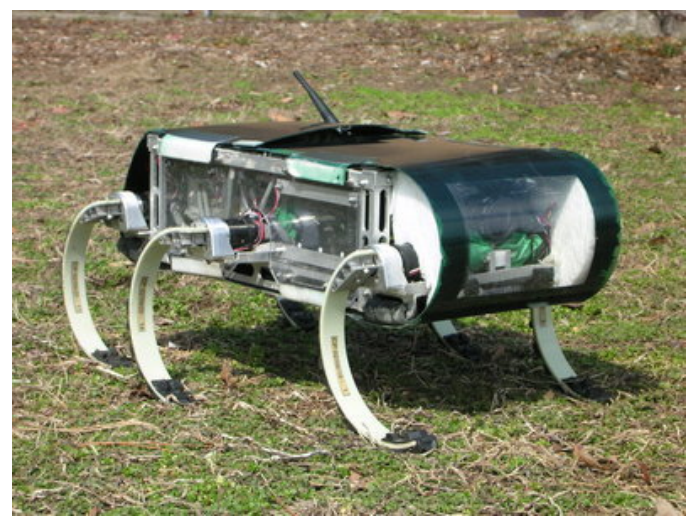

Figura 2: Robot RHex

Numerosos estudios han analizado la dinámica de este tipo de robots, desde la aplicación del modelo del péndulo invertido [8] [9] hasta modelos de patas más generalistas [12].

A pesar de la gran proliferación de este tipo de robots, no se han realizado estudios que profundicen en el estudio cinemático de las patas de los mismos [6], [10]. Sin embargo, este estudio es fundamental para este tipo de robots, condicionando el proceso de diseño y construcción de los mismos.

En este artículo se demuestra el desarrollo matemático necesario para calcular el desplazamiento del robot y cuyos resultados han sido validados mediante simulaciones con un resultado correcto.

La estructura del artículo comienza con esta pequeña introducción, para continuar con el análisis matemático del modelo. Posteriormente, se procede a la explicación de las simulaciones realizadas y, por último, se encuentran los apartados de resultados y conclusiones.

\section{ANÁLISIS MATEMÁTICO}

En esta sección se detalla el procedimiento matemático desarrollado. En la primera parte se hace el estudio con un única pata, y en la segunda parte se extrapolan los cálculos al sistema robótico compuesto por seis patas.

Las hipótesis tomadas para el desarrollo matemático han sido las siguientes:
No hay deslizamiento: Cada pata rueda sin deslizar sobre el suelo, y el chasis del robot tampoco desliza al tocar el suelo. Hay que tener en cuenta que no se considera deslizamiento ni en la dirección transversal ni en la longitudinal. Esta hipótesis se puede aceptar válida en los modos de marcha de las 6 patas a la vez y en el trípode alterno. En otros modos de marcha considerados para este robot (tetrápoda y onda por ejemplo) hay deslizamiento en las dirección longitudinal y/o transversal.

Sólido rígido: Todos los elementos del robot se consideran indeformables.

Pata circular: El contorno exterior e interior de la pata son dos círculos concéntricos.

\subsection{CINEMÁTICA DE UNA PATA INDIVIDUAL}

Como se ha comentado anteriormente, en esta sección se va a analizar la cinemática de una pata individual, considerando que en su movimiento no influyen las demás patas. Esto sólo es cierto para el modos de marcha tipo onda completa que se explica en [10]. Posteriormente, se extenderá este modelo al modo de marcha denominado como trípode alterno mediante el cual se suelen desplazar la gran mayoría de los hexápodos.

Para ello se va a imponer la condición inicial de que el robot se encuentre apoyado sobre una superficie totalmente lisa. Bajo esta premisa, el eje de la pata va a describir una curva perteneciente a la familia de los trocoides.

El trocoide es aquella curva que describe un punto asociado a una circunferencia que rueda sin deslizar. Esta circunferencia se conoce como circunferencia generatriz.

Las ecuaciones paramétricas que definen un trocoide son las siguientes:

$$
\begin{aligned}
x & =a \theta-b \cdot \cos \theta \\
y & =a-b \cdot \cos \theta
\end{aligned}
$$

En general, los trocoides pueden ser de tres tipos (figura 3):

Cicloide ordinario El punto pertenece a la circunferencia generatriz $(b=a)$.

Cicloide acortado El punto está en el interior de la circunferencia generatriz $(b<a)$.

Cicloide alargado El punto está fuera de al circunferencia generatriz $(b>a)$. 


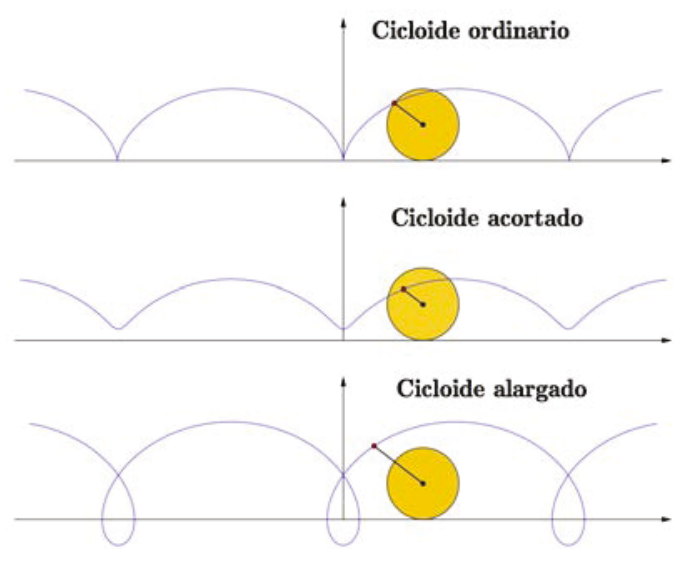

Figura 3: Familia de trocoides

En el momento en que la pata entra en contacto con el suelo se obtienen las siguientes igualdades que describen el sistema, cuya referencia puede observarse en las figuras 4 y 5 :

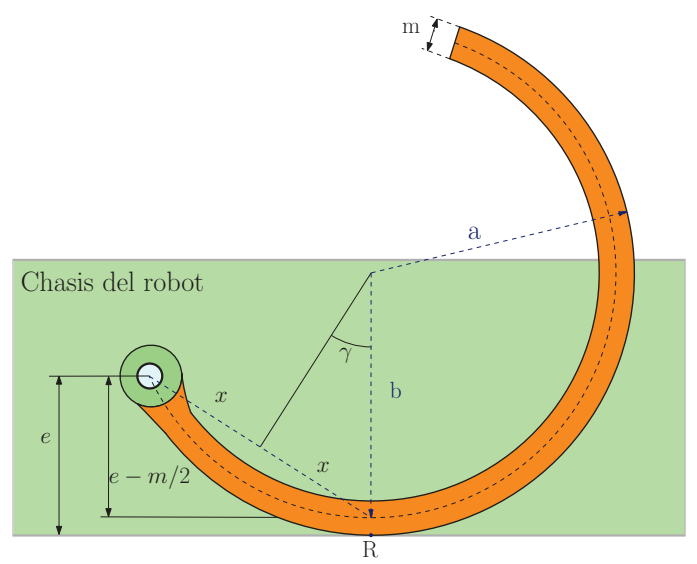

Figura 4: Pata apoyada

$$
\begin{aligned}
\tan \gamma & =\frac{e-\frac{m}{2}}{2 x} \\
\sin (\gamma) & =\frac{x}{b} \\
m & =2(a-b) \\
\alpha & =\beta-2 \gamma \\
c^{2} & =(a+b \cos (\beta-\pi))^{2}+(b \sin (\beta-\pi))^{2}
\end{aligned}
$$

A partir de estas ecuaciones, se pueden despejar las variables $x, \gamma, m, \alpha, c$.

Para obtener una descripción completa del sistema el movimiento que describe la pata respecto a su eje se va a descomponer en dos tramos:

Tramo 1: Tramo A-B en la figura 6. Es el descrito por un cicloide acortado y es cuando la pata está en contacto físico con el suelo.

Tramo 2: Tramo B-C en la figura 6. Es el descrito por el arco de una circunferencia y es

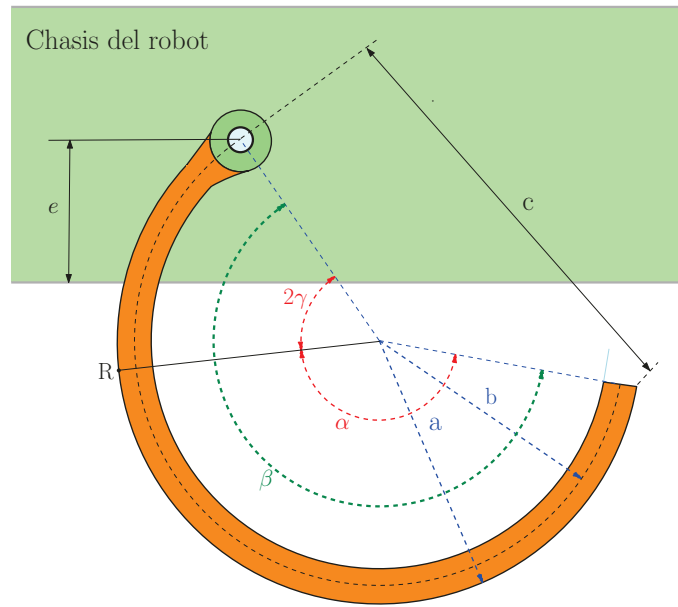

Figura 5: Pata levantada

cuando la pata no está en contacto físico con el suelo.

$\left\{\begin{array}{l}\text { Tramo } 1\left\{\begin{array}{l}x=a \theta-b \sin (\theta) \\ y=a-b \cos (\theta)\end{array}, \theta \in\left[\theta_{i}, \theta_{f}\right]\right. \\ \text { Tramo 2 }\left\{\begin{array}{l}x=c \cos \left(\theta^{\prime}-\delta_{1}\right)+x_{0} \\ y=-c \sin \left(\theta^{\prime}-\delta_{1}\right)\end{array}, \theta^{\prime} \in\left[0, \theta_{f}^{\prime}\right]\right.\end{array}\right.$

donde:

$$
\begin{aligned}
\theta_{i} & =2 \gamma \\
\theta_{f} & =\alpha+2 \gamma \\
\theta_{f}^{\prime} & =\delta_{1}-\delta_{2} \\
\delta_{1} & =\operatorname{atan}\left(\frac{a-b \cos (\alpha)}{(a \alpha-b \sin \alpha)-x_{0}}\right) \\
\delta_{2} & =\operatorname{atan}\left(\frac{e}{c}\right) \\
x_{0} & =b \sin \left(\theta_{i}\right)+a\left(\theta_{f}-\theta_{i}\right)+a \theta_{i}-b \sin \left(\theta_{i}\right)=a \theta_{f}
\end{aligned}
$$

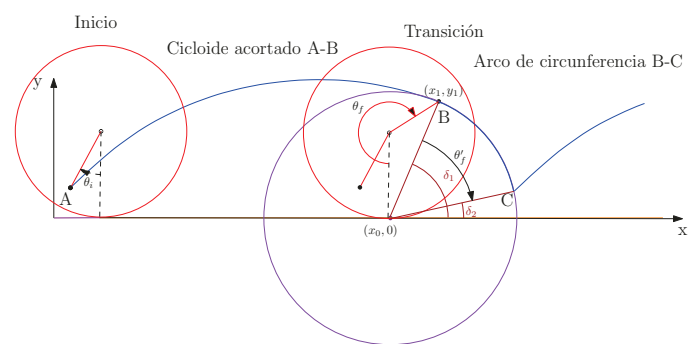

Figura 6: Curvas en el movimiento

En la figura 7 se puede ver el movimiento completo de la pata. La longitud recorrida por la pata en un ciclo puede obtenerse mediante la ecuación 4, donde todos los datos son conocidos:

$l_{\text {ciclo pata individual }}=a\left(\theta_{f}-\theta_{i}\right)+b \sin \left(\theta_{i}\right)+c \cos \left(\delta_{2}\right)$ 


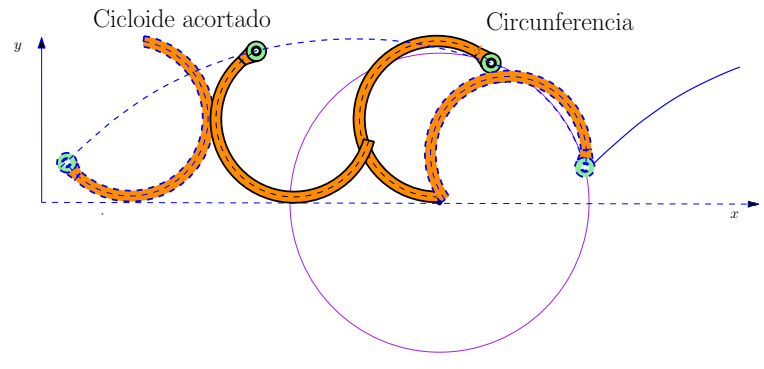

Figura 7: Movimiento completo

\subsection{CINEMÁTICA DEL ROBOT COMPLETO}

A continuación se va a abordar el desarrollo matemático para el conjunto del robot con sus seis patas.

Si las seis patas se moviesen a la vez y sin ningún desfase entre ellas, la cinemática del robot completo sería idéntica a la desarrollada para una pata individual.

En el modo de marcha llamado trípode alterno primero se mueven las patas derechas delantera y trasera junto con la pata central izquierda y posteriormente las otras 3. En este modo de marcha, hay que tener en cuenta que la serie de movimientos descritos anteriormente no se van a describir de forma completa, sino sólo una parte de los mismos.

Asimismo, en el trípode alterno, la curva que va a describir el eje de rotación de la pata va a depender de si se llega o no a realizar parte del arco de la circunferencia. Esto a su vez dependerá del parámetro $\lambda$ (ver figuras 8 y 10). Este parámetro es escogido a elección del programador del robot, cumpliéndose la siguiente ecuación:

$$
h=a+b \sin \left(\lambda-\frac{\pi}{2}\right)
$$

Donde $h$ es la altura mínima a la que estará el chasis del robot durante la ejecución de este modo de marcha. Por lo tanto, el modelado del movimiento puede ser formulado de dos formas distintas según:

- Si se hace parte del arco de circunferencia: Esto se da si $h<c \sin \left(\delta_{1}\right)$. En este caso, la curva es la siguiente:

$$
\left\{\begin{array}{l}
\text { Tramo } 1\left\{\begin{array}{l}
x=a \theta-b \sin (\theta) \\
y=a-b \cos (\theta)
\end{array}, \theta \in\left[\theta_{i}, \theta_{f}\right]\right. \\
\text { Tramo } 2\left\{\begin{array}{l}
x=c \cos \left(\theta^{\prime}-\delta_{1}\right)+x_{0} \\
y=-c \sin \left(\theta^{\prime}-\delta_{1}\right)
\end{array}, \theta^{\prime} \in\left[0, \theta_{f}^{\prime}\right]\right.
\end{array}\right.
$$

donde:

$$
\begin{aligned}
& \theta_{i}=\lambda \\
& \theta_{f}=\alpha+2 \gamma
\end{aligned}
$$

y $\theta_{f}^{\prime}$ se halla haciendo $h=-c \sin \left(\theta^{\prime}-\delta_{1}\right)$.

Y por tanto, la longitud recorrida queda como la suma de la parte de la cicloide más la suma de la parte del arco de circunferencia:

$l_{\text {ciclo tripode }}=$ Cicloide + Circunferencia

$\left\{\begin{array}{l}\text { Cicloide }=a\left(\theta_{f}-\theta_{i}\right)+b\left(\sin \theta_{i}-\sin \theta_{f}\right) \\ \text { Circunferencia }=\cos \left(\theta_{f}^{\prime}-\delta_{1}\right)-c \cos \left(\delta_{1}\right)\end{array}\right.$

Por lo que al final se obtiene la siguiente expresión que define la longitud recorrida:

$$
\begin{gathered}
l_{\text {recorrida }}=\sum_{i=1}^{n} l_{\text {ciclotripode }}=n\left(a\left(\theta_{f}-\theta_{i}\right)+\right. \\
\left.b\left(\sin \theta_{i}-\sin \theta_{f}\right)+c \cos \left(\theta_{f}^{\prime}-\delta_{1}\right)-c \cos \left(\delta_{1}\right)\right)
\end{gathered}
$$

- Si no se hace parte del arco de circunferencia: Esto se da si $h>c \sin \left(\delta_{1}\right)$. En este caso sólo se tiene un tramo:

$$
\left\{\begin{array}{l}
x=a \theta-b \sin (\theta) \\
y=a-b \cos (\theta)
\end{array}, \theta \in\left[\theta_{i}, \theta_{f}\right]\right.
$$

donde:

$$
\theta_{i}=\lambda
$$

$\theta_{f}$ se halla haciendo $h=a-b \cos \left(\theta_{f}\right)$

La longitud recorrida será:

$$
l_{\text {ciclo tripode }}=\underbrace{a\left(\theta_{f}-\theta_{i}\right)+b\left(\sin \theta_{i}-\sin \theta_{f}\right)}_{\text {Cicloide }}
$$

y por consiguiente:

$$
\begin{array}{r}
l_{\text {recorrida }}=\sum_{i=1}^{n} l_{\text {ciclo tripode }}=n\left(a\left(\theta_{f}-\theta_{i}\right)+\right. \\
\left.b\left(\sin \theta_{i}-\sin \theta_{f}\right)\right)
\end{array}
$$

A partir de la parametrización anterior, se puede hallar la velocidad durante el tramo del cicloide en función del parámetro $\theta$ :

$$
\left\{\begin{array}{r}
\dot{x}=-b \cos (\theta) \dot{\theta} \\
\dot{y}=b \sin (\theta) \dot{\theta}
\end{array}\right.
$$

Donde $\dot{\theta}$ es la velocidad del eje del motor en ese momento. Si $\dot{\theta}$ es constante (como ocurre en la simulación posteriormente explicada) es trivial demostrar que el módulo de la aceleración será constante:

$$
|\vec{a}|=\sqrt{\ddot{x}^{2}+\ddot{y}^{2}}=b \dot{\theta}^{2}
$$

Lógicamente, durante el tramo del arco de circunferencia, si $\dot{\theta}$ es constante, el módulo de las velocidad y de la aceleración también serán constantes. 


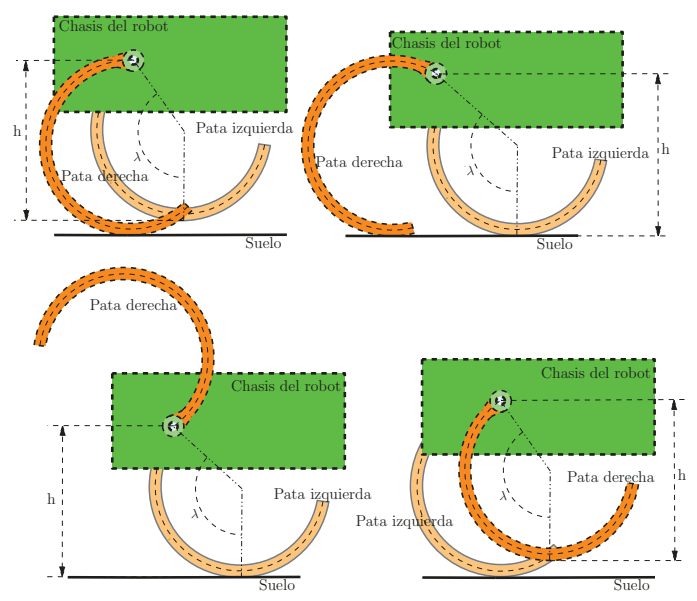

Figura 8: Movimiento en el trípode alterno

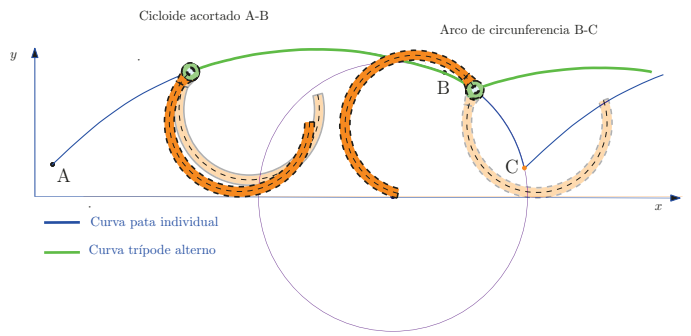

Figura 9: Comparación trípode alterno y pata individual

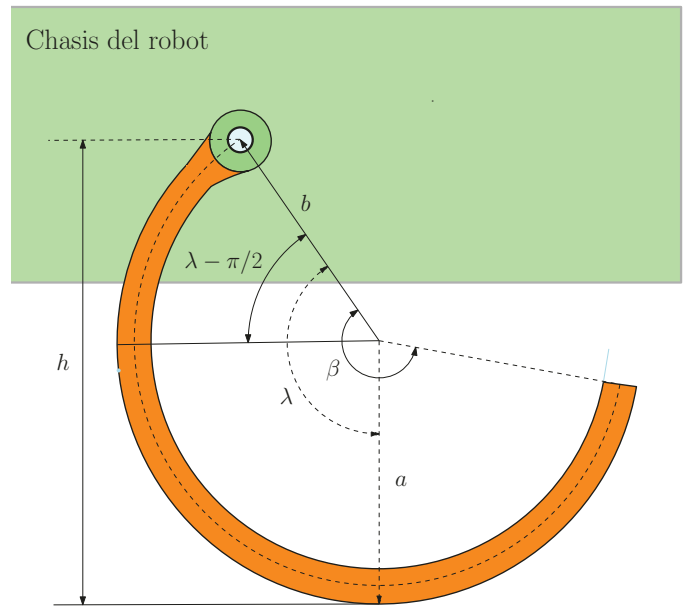

Figura 10: Variables en el trípode alterno

\section{SIMULACIONES REALIZADAS}

Para validar el modelo cinemático realizado, se ha llevado a cabo una simulación dinámica con Autodesk Inventor@. Es importante destacar que en esta simulación se han impuesto las mismas hipótesis consideradas en el modelo matemático: No hay deslizamiento, y los sólidos se consideran como sólidos rígidos. La hipótesis de pata circular se ha impuesto con el diseño 3D de la pata.

Tanto para el caso de pata individual como el caso del trípode alterno, se ha usado el modelo que se puede ver en la figura 11 para simular y verificar el modelo cinemático. Este modelo consta únicamente de dos patas, que unido a la restricción del cuerpo a girar, produce el mismo movimiento cinemático realizado por el robot de seis patas. Esta simplificación del número de patas (que no afecta en nada al resultado cinemático) es debida a reducir los tiempos de simulación, los cuales aumentan considerablemente con el número de patas del modelo.

Los parámetros del modelo usado han sido $a=$ $85,8 \mathrm{~mm}, m=10.8 \mathrm{~mm}, \beta=203.4^{\circ}$ y $e=$ $48.3 \mathrm{~mm}$.

Además, todas las posiciones, velocidades y aceleraciones resultantes de la simulación se han tomado en el eje de la pata.

En la figura 12 se puede observar como se han ajustado los parámetros para producir el movimiento del robot.

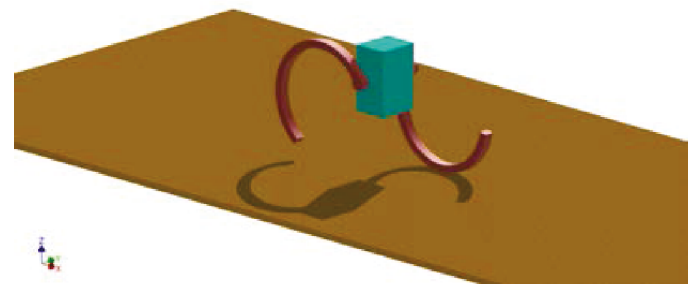

Figura 11: Modelo usado

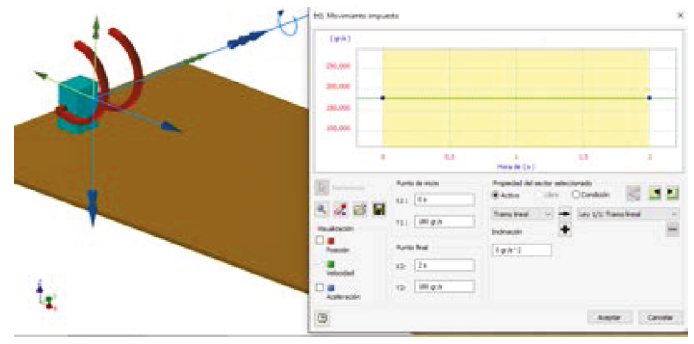

Figura 12: Movimiento impuesto a los ejes de las ruedas 


\subsection{SIMULACIÓN PARA UNA PATA INDIVIDUAL}

En primer lugar se ha realizado la simulación de una pata individual (o lo que sería lo mismo, un desplazamiento en el que todas las patas se mueven a la vez y sin desfases).

A cada eje de las patas del modelo se le ha configurado una velocidad angular constante e igual a $180 \mathrm{grados} / \mathrm{segundo}(\pi \mathrm{rads} / \mathrm{s})$. Además, se ha simulado una secuencia de dos ciclos seguidos.

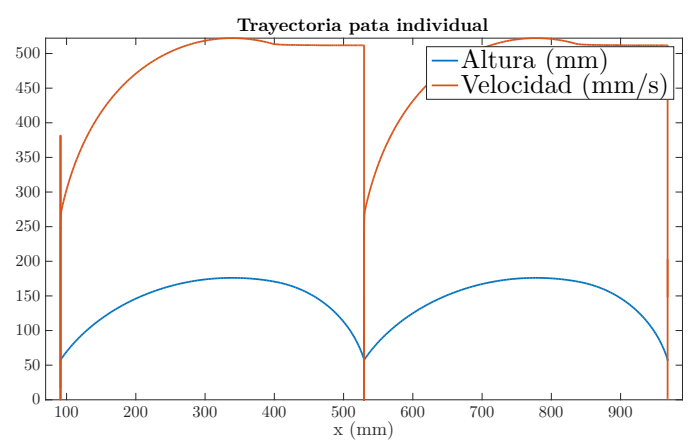

Figura 13: Altura y Velocidad del eje de la pata en función del tiempo

En la figura 13 se muestra la gráfica que compara la altura con la velocidad de giro del eje en función del tiempo. Se puede apreciar cómo la altura del eje describe un cicloide acortado al principio, para posteriormente describir un arco de circunferencia, tal y como se había predicho con el modelo cinemático teórico desarrollado en la sección 2.1. Asimismo, se puede ver cómo en el momento en el que el punto del eje de la pata comienza a describir una circunferencia, el módulo de la velocidad total se hace constante.

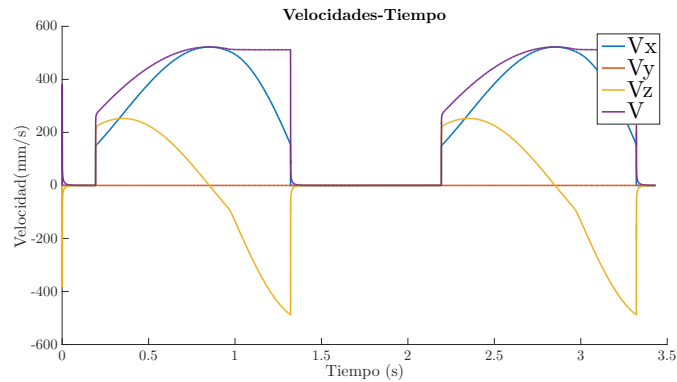

Figura 14: Velocidades del eje de la pata en función del tiempo

En la figura 14 se puede apreciar una gráfica con las velocidades en los distintos ejes, así como el módulo de la velocidad total. La velocidad $V_{y}$ es nula debido a que el robot se esta desplazando en el plano $X Z$.

En la figura 15 se puede apreciar la gráfica de las aceleraciones en función del tiempo. Se puede com-

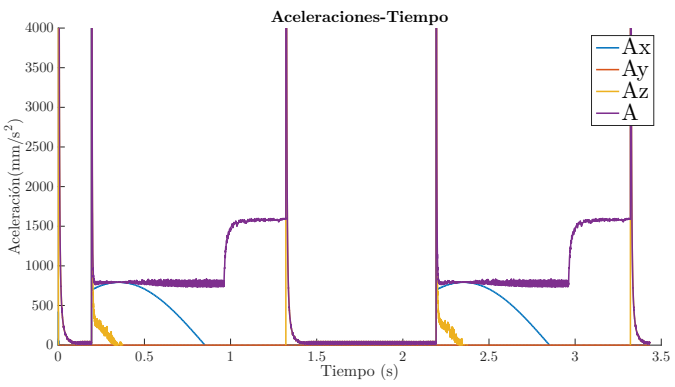

Figura 15: Aceleraciones del eje de la pata en función del tiempo

probar que la aceleración es constante tanto en el tramo del cicloide como en el tramo de la circunferencia. El ruido que se puede ver en la figura 15 se debe principalmente al método que emplea Inventor para calcular la aceleración del eje de la pata (por derivaciones sucesivas a partir de la posición), lo que provoca una acumulación de errores numéricos. Asimismo, es importante destacar el pico de aceleración que presenta al iniciar la marcha, ya que se le exige que pase de estar parado a estar moviéndose en tiempo cero.

Por último, en la figura 16 se puede observar la simulación realizada con la trayectoria seguida por el eje de la pata.

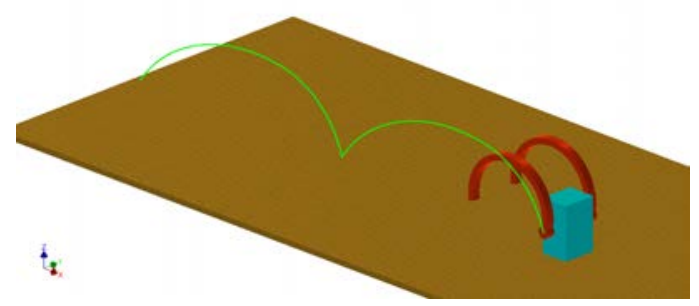

Figura 16: Trayectoria seguida por el eje de la pata

\subsection{SIMULACIÓN PARA EL ROBOT COMPLETO}

En esta sección se muestran los resultados de la simulación realizada para el modo de marcha denominado trípode alterno. Para ello, una pata se ha configurado con una velocidad constante de 180 grados $/$ segundo, y la otra se ha dejado fija en una misma posición. El parámetro $\lambda$ se ha escogido con un valor de $123.4^{\circ}$, de forma que (y teniendo en cuenta los parámetros de la pata) el eje de la pata va a describir tanto el cicloide como parte del arco de circunferencia.

Las figuras 17, 18 y 19 son gráficas obtenidas en esta simulación.

Concretamente, en la figura 17 se pude apreciar el tramo en el cual el módulo de la velocidad se hace 
constante (correspondiente al tramo en el cual el punto del eje describe una circunferencia).

En la figura 18 se puede destacar que la velocidad en la dirección del eje $\mathrm{z}$ se hace negativa justo cuando el chasis empieza a descender (y por tanto, justo cuando la altura del chasis de la gráfica 17 ha alcanzado su máximo).

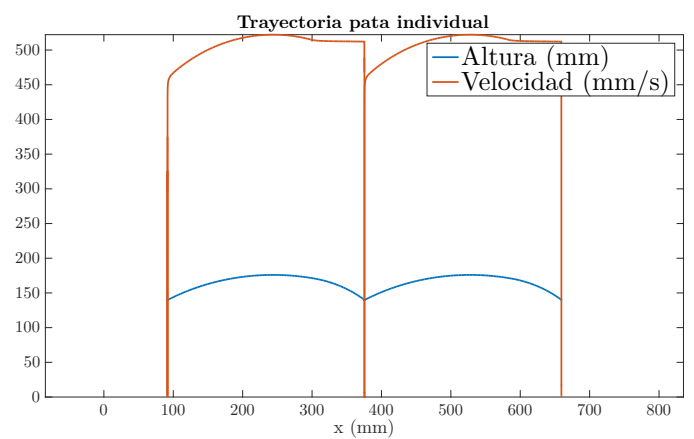

Figura 17: Altura y Velocidad del eje de la pata en función del tiempo

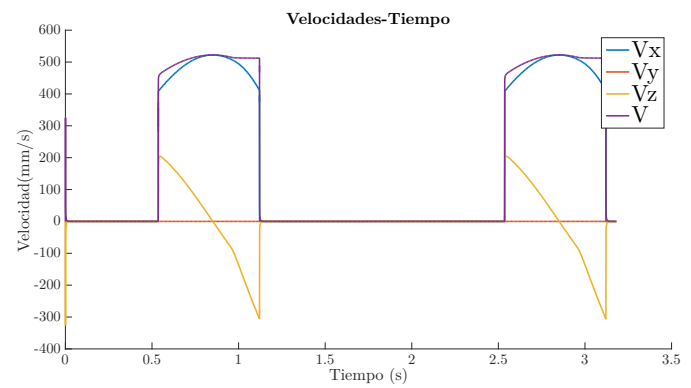

Figura 18: Velocidades del eje de la pata en función del tiempo

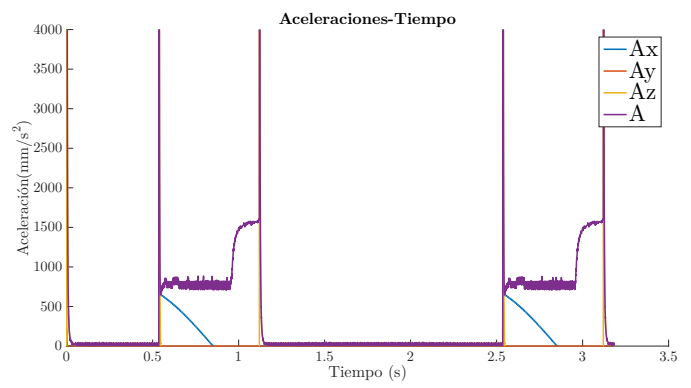

Figura 19: Aceleraciones del eje de la pata en función del tiempo

En la figura 19, de la misma manera que pasaba en la figura15, los picos en la aceleración son debidos a que se le exige al modelo un incremento de velocidad finito en un tiempo cero.

\section{RESULTADOS}

Se ha desarrollado un modelo matemático de la cinemática del robot, tanto de una pata individual

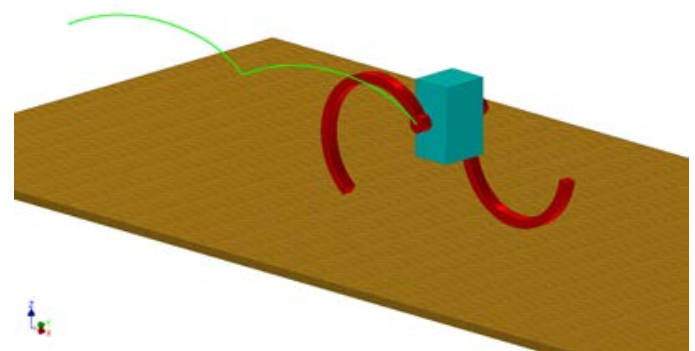

Figura 20: Trayectoria seguida por el eje

como del robot completo.

En el caso de una pata individual, ésta describe una curva que es la suma de un arco de cicloide y un arco de circunferencia. Por otro lado, para el robot completo, depende del modo de marcha. $\mathrm{Si}$ el robot mueve todas las patas a la vez y sin desfase, el modelo cinemático coincide con el de la pata individual. Si el modo de marcha es el trípode alterno, se ha demostrado que la curva descrita es parte de la curva descrita por la pata individual.

Para la comprobación del modelo matemático, se han realizado una serie de simulaciones cinemáticas del robot, de las cuales se han extraído las gráficas de posiciones, velocidades y aceleraciones. Los resultados obtenidos en las simulaciones coinciden en su totalidad con el modelo matemático desarrollado en la primera parte.

Los resultados de este análisis cinemático y de la simulación tienen su aplicabilidad práctica en los siguientes aspectos: odometría, criterios de diseño y funcionalidades extras.

La odometría es su aplicación más directa, permitiendo que el robot conozca su posición a partir de la velocidad impuesta en sus motores. La fusión sensorial de esta odometría con otros sensores (GPS, IMU,...) y la aplicación de técnicas de fusión sensorial (filtro de Kalman por ejemplo) permitirían conocer al robot su posición en todo momento.

Por otro lado, los resultados obtenidos permiten elegir tanto las dimensiones de la pata como el parámetro $\lambda$ de acuerdo con la variación de altura y la altura máxima deseada.

En cuanto a la variación de altura del chasis, en el modo de marcha trípode alterno es necesario saber a priori este valor, puesto que influye directamente en la estabilidad. Asimismo, es un parámetro a considerar a la hora de seleccionar los sensores (infrarrojos, cámaras, láser,...). Estos sensores son imprescindibles sobre todo cuando la aplicación de estos robots son las tareas de búsqueda y rescate [7]. Asimismo, la altura máxima a la que va a estar el chasis del robot es esencial en el di- 
seño del mismo, pues condiciona la altura mínima de los huecos o agujeros a través de los cuales el robot podrá pasar.

La variación de altura descrita en el párrafo anterior permite dotar al robot de funcionalidades extras como podría ser la de escanear una zona con un láser 2D sin necesidad de una unidad pantilt $[6]$.

\section{CONCLUSIONES}

Este artículo realiza un análisis detallado del modelado cinemático del sistema de locomoción de los robots hexápodos con patas en forma de C.

Hasta la fecha no se conoce ningún estudio en el que se desarrolle una formulación matemática en la que se tengan en cuenta todo el conjunto de variables del sistema para poder definir correctamente el avance del robot.

Asimismo, el modelado matemático realizado cuadra perfectamente con los resultados obtenidos mediante simulación, lo cual valida el modelo desarrollado.

Es por ello que este artículo puede considerarse una buena base para el estudio de los distintos modos de locomoción que pueden generar este tipo de robots y sus aplicaciones relacionadas, tal y como se describen en el apartado anterior.

\section{Agradecimientos}

Esta investigación ha recibido fondos del proyecto RoboCity2030-III-CM (Robótica aplicada a la mejora de la calidad de vida de los ciudadanos. fase III; S2013/MIT-2748), financiado por los Programas de Actividades I+D en la Comunidad de Madrid y los Fondos Estructurales de la Unión Europea, y del proyecto DPI2014-56985-R (Protección robotizada de infraestructuras críticas), financiado por el Ministerio de Economía y Competitividad del Gobierno de España.

\section{Referencias}

[1] M. Nowicki A. Roennau, G. Heppner and R. Dillmann. Lauron v: A versatile six-legged walking robot with advanced maneuverability. IEEE ASME, pages 82-87, July 2014.

[2] R. Balasubramanian. Legless locomotion: Concept and analysis. Technical report, Citeseer, 2004.

[3] T. Zhang C. Li and D. Goldman. A terradynamics of legged locomotion on granular media. Science, 339(6126):1408-1412, 2013.
[4] X. Pacheco C. Nie and M. Spenko. Robots on the move: versatility and complexity in mobile robot locomotion. IEEE Robotics \& Automation, 20(4):72-82, 2013.

[5] L. R. Palmer III, E. D. Diller, and R. D. Quinn. Design of a wall-climbing hexapod for advanced maneuvers. IEEE IROS, pages 625-630, 2009.

[6] E. Z. Moore. Leg design and stair climbing control for the rhex robotic hexapod. Master's thesis, Department of Mechanical Engineering McGill University, January 2002.

[7] Robin Murphy. Disaster Robotics. MIT Press, 2014.

[8] H. Komsuoglu D. Koditschek H. Brown Jr M. Buehler N. Moore D. McMordie R. Altendorfer, U. Saranli and R. Full. Evidence for spring loaded inverted pendulum running in a hexapod robot. Springer, 2001.

[9] H. Komsuoglu D. Koditschek H. Brown Jr M. Buehler N. Moore D. McMordie R. Altendorfer, U. Saranli and R. Full. Rhex: a biologically inspired hexapod runner. Autonomous Robots, 11(3):207-213, 2001.

[10] Jorge De León Rivas. Definición y análisis de los modos de marcha de un robot hexápodo para tareas de búsqueda y rescate. Master's thesis, Universidad Politécnica de Madrid, October 2015.

[11] B. Siciliano and O. Khatib. Springer handbook of robotics. Springer, 2008.

[12] M. Buehler U. Saranli and D. Koditschek. Rhex: A simple and highly mobile hexapod robot. The International Journal of Robotics Research, 20(7):616-631, 2001. 